
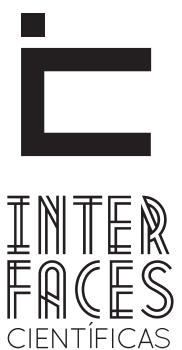

SAÚDE E AMBIENTE

ISSN IMPRESSO 2316-3313

E - ISSN 2316-3798

DOI - 10.17564/2316-3798.2017v5n3p 77-86

\title{
COMUNICAÇÃO E HUMANIZAÇ̃̃O: FERRAMENTAS DA ENFERMAGEM NA ASSISTÊNCIA À FAMILLAA DO PACIENTE ONCOLÓGICO
}

\author{
COMMUNICATION AND HUMANIZATION: NURSING TOOLS IN PATIENT CARE FAMILY ONCOLOGICAL \\ COMUNICACIÓN Y HUMANIZACIÓN: HERRAMIENTAS DE ENFERMERía EN LA ASISTENCIA A LA FAMILLA DEL PACIENTE ONCOLÓOICO
}

Carlos Sebastião Oliveira Martins ${ }^{1}$

Damião da Conceição Araújo $^{3}$

Maria Inês Brandão Bocardi ${ }^{5}$
Heline Rocha de Andrade 2

Fernanda Gomes de Magalhães Soares Pinheiro ${ }^{4}$

Andréia Centenaro Vaez ${ }^{6}$

\section{RESUMO}

As neoplasias constituem um problema de saúde pública no cenário mundial, destaca-se que a assistência deve ser orientada para o paciente e família, os quais são vulneráveis e requerem uma assistência que integre os aspectos biológicos, psicológicos e espirituais, portanto a comunicação e humanização são ferramentas essenciais nesse processo. Os objetivos do estudo foram: analisar o impacto no contexto familiar do paciente com diagnóstico de câncer e identificar a percepção da família em relação a comunicação e o cuidado humanizado do enfermeiro. Trata-se de um estudo descritivo e qualitativo, realizado com oito famílias de pacientes oncológicos internados no Centro de Oncologia Osvaldo Leite em Sergipe, no ano de 2011. Dados qualitativos foram submetidos à análise categorial subsidiados nos conceitos de Bardin, que identificaram duas categorias: impacto da doença fora da possibilidade terapêutica de cura no contexto familiar e Importância da humanização e comunicação na assistência de enfermagem. Evidenciou-se que o perfil sociodemográfico dos entrevistados era: sexo feminino, casado, católico, com ensino funda- 
mental incompleto. Das categorias e análise dos conteúdos, destacou-se que os familiares se mostravam amedrontados, confusos e abalados diante do câncer e a grande maioria não sabiam reconhecer quem era o enfermeiro, que caracteriza um desvio na comunicação. Conclui-se que a assistência do enfermeiro, realizada com uma comunicação adequada e humanizada, produz melhor qualidade de vida, reduz angús-

\section{ABSTRACT}

Neoplasms constitute a public health problem on the world stage, which is a care must be patient and family oriented, banks are vulnerable and require assistance that integrates the biological, psychological and spiritual aspects, therefore, communication and They are important tools in this process. In view of the above, the objectives of the study were: to analyze the impact in the family context of the patient with diagnosis of cancer and identify a perception of the family in relation to communication and the humanized care of the nurse. This is a descriptive and qualitative study carried out with eight families of cancer patients hospitalized at the Osvaldo Leite Oncology Center in Sergipe, in the year 2011. Qualitative data submitted to categorical analysis subsidized in the concepts of Bardin, which identified two categories: Impact Of the disease to a therapeutic possibility of cure not family context and Importance of humanization and com-

\section{RESUMEN}

Neoplasias constituyen un problema de salud pública en la escena del mundo, se resalta que la asistencia es un servicio debe ser dirigida al paciente y su familia, los bancos son vulnerables y requieren de apoyo que integre lo biológico, psicológico y espiritual, por lo tanto, la comunicación y la humanización son herramientas importantes en este proceso. Teniendo en cuenta lo anterior, los objetivos del estudio fueron tias, medos e proporciona ao paciente e família, maior conforto e segurança durante o internamento.

\section{PALAVRAS-CHAVES}

Humanização da Assistência, Comunicação, Enfermagem familiar, Enfermagem Oncológica.

munication in nursing care. It was evidenced that the sociodemographic profile of the interviewees was: female, married, Catholic, with incomplete elementary school. The categories and analysis of the contents, highlighting that the relatives are shown frightened, confused and shaken by cancer and a large did not know who the nurse was, which characterize a deviation in communication. It is concluded that nursing care, performed with a quality and humanized evaluation, produces a better quality of life, reduced distress, fears and offers the patient and family greater comfort and safety during hospitalization.

\section{KEYWORDS}

Humanization of Assistance, Communication, Family Nursing, Oncology Nursing. evaluar el impacto en el contexto familiar del paciente diagnosticado con cáncer e identificar la percepción de la familia en relación con la comunicación y el cuidado humanizado del enfermero. Se trata de un estudio descriptivo y cualitativo, realizado con ocho familias de los pacientes con cáncer en el Centro de Oncología Osvaldo Leite Sergipe, en 2011. Los datos cualitativos han sido sometidos a análisis de catego- 
rías en conformidad con los conceptos de Bardin, que identificó dos categorías: Impacto de la enfermedad a parte del contexto de curación posibilidad terapéutica desconocida y la importancia de la humanización y la comunicación en la atención de enfermería. Se hizo evidente que perfil sociodemográfico de los encuestados: mujer, casado, católico, con educación primaria incompleta. De las categorías y análisis de los contenidos, especialmente la familia se muestran asustados, confusos y agitados antes de que el cáncer y grandes no sabían reconocer quién era la enfermera, lo que demostraba un desvío en la comunicaci-

\section{INTRODUÇ̃̃̃O}

As neoplasias caracterizam-se como um problema de saúde pública e modificam o perfil de morbidade e mortalidade dos indivíduos acometidos (SILVA et al., 2012). 0 envelhecimento progressivo da população brasileira, a urbanização e o diagnóstico tardio da grande maioria dos cânceres, proporciona o crescimento dos portadores de doenças crônicas de caráter terminal (BRASIL, 2015).

Os avanços tecnológicos na área de biociências têm favorecido a sobrevivência de pacientes, o que provoca um aumento significativo do número de internações hospitalares de pacientes em fase terminal, para a realização de cuidados paliativos (BRASIL, 2015). No Brasil, o câncer é a segunda causa de mortalidade da população, representando $17 \%$ dos óbitos de causa conhecida (PINTO et al., 2011).

A estimativa, no biênio 2016-2017, aponta a ocorrência de cerca de 600 mil casos novos de câncer no país. 0 perfil epidemiológico observado assemelha-se ao de outros países da América Latina e Caribe, onde os cânceres de próstata (61 mil) em homens e mama (58 mil) em mulheres serão os mais incidentes. Além disso, os tipos mais frequentes de câncer em homens serão os de próstata (28,6\%), pulmão $(8,1 \%)$, intestino $(7,8 \%)$, estômago $(6,0 \%)$ e cavidade oral $(5,2 \%)$. ón. Llegamos a la conclusión de que un cuidado de enfermería, realizada con la evaluación de calidad y humanizada, produce una mejor calidad de vida, reducción de la ansiedad, miedos y ofrece al paciente y su familia, la comodidad y la seguridad durante la hospitalización

\section{PALABRAS CLAVE}

Humanización de la Asistencia, Comunicación, Enfermería Familiar, Enfermería Oncológica.

Nas mulheres, os cânceres de mama (28,1\%), intestino $(8,6 \%)$, colo do útero (7,9\%), pulmão $(5,3 \%)$ e estômago (3,7\%) serão os principais (BRASIL, 2015).

O Ministério da Saúde divulgou no ano de 2005, a Política Nacional de Atenção Oncológica, que contempla ações de promoção, prevenção, diagnóstico, tratamento, reabilitação e cuidados paliativos, implantada em todas as unidades federadas. Portanto, deve ser organizado para atender as necessidades dos pacientes, gerar qualidade de vida e conforto em cada etapa do tratamento (BRASIL, 2005).

A família sofre impacto significativo quando um membro recebe o diagnóstico de câncer, esse deve ser reconhecido pelo enfermeiro, para melhorar a assistência e inserir a família no plano de cuidado de enfermagem (OLIVEIRA et al., 2014).

A comunicação e humanização são ferramentas primordiais no contexto da assistência a saúde, em especial no exercício profissional do enfermeiro, que deve prestar assistência aos pacientes oncológicos e familiares. Quando utilizadas de maneira adequada, possibilitam um relacionamento terapêutico efetivo, acolhimento e resolutividade dos problemas emergentes (RODRIGUES; FERREIRA; MENEZES, 2010; PETERSON; CARVALHO, 2011). 
Mediante a problemática, os objetivos do estudo foram: analisar o impacto no contexto familiar do paciente com diagnóstico de câncer e identificar a percepção da família em relação à comunicação e o cuidado humanizado do enfermeiro.

\section{METODOLOGIA}

Trata-se de um estudo descritivo com abordagem qualitativa, na modalidade de análise temática subsidiada em Bardin (1979). Realizado no centro de oncologia Osvaldo Leite, localizado no Hospital de Urgências de Sergipe (HUSE), no município de Aracaju-SE, no ano de 2011. Realizado com oito famílias de pacientes oncológicos em cuidados paliativos, com base nos seguintes critérios de inclusão: maior de 18 anos, possibilitado de comunicar-se verbalmente e os critérios de exclusão: portadores de problemas psiquiátricos ou déficit cognitivo.

A pesquisa foi aprovada pelo comitê de ética em pesquisa (CEP) da Universidade Tiradentes, sob o parecer $n^{\circ} 080411 R$. Todos os direitos e identidade dos voluntários foram resguardados e atenderam a Resolução n 466/2012, do Conselho Nacional de Saúde do Ministério de Saúde. Os participantes assinaram o termo de consentimento livre e esclarecido e o termo de cessão de direitos para uso da voz. Para manter o sigilo dos entrevistados, foi realizada a codificação das entrevistas por meio da utilização da letra "E".

A coleta de dados foi realizada durante sete dias e utilizou-se como instrumento um formulário composto por perguntas abertas e fechadas elaboradas pelos pesquisadores, dividido em dados sociodemográficos e cinco questões norteadoras. As variáveis independentes foram Humanização e Comunicação e, as variáveis dependentes: medo, ansiedade e angústias da família. As entrevistas foram gravadas, para garantir fidedignidade e transcritas na íntegra, a fim de garantir a linguagem, conteúdo e sentido conferidos pelo interlocutor.

Foi realizada a análise descritiva dos dados sociodemográficos. Os dados qualitativos foram subsidiados nos conceitos de Bardin e compreenderam as etapas operacionais de pré-análise, categorização e tratamento dos dados. Na etapa da pré-análise foram realizadas as leituras dos conteúdos a partir da leitura dirigida das entrevistas transcritas, nesta etapa foram apresentados os conteúdos de forma exaustiva a fim de identificar a representatividade, homogeneidade e pertinência.

Durante a categorização explorou-se o material, com transformação dos resultados brutos em categorias significativas, sendo elas: 0 impacto da doença fora da possibilidade terapêutica de cura no contexto familiar e Importância da humanização e comunicação na assistência de enfermagem ao paciente oncológico e seus familiares. Na etapa do tratamento dos dados foram apresentados a partir das falas dos sujeitos entrevistados.

\section{RESULTADOS}

A análise descritiva das oito famílias evidenciou que o perfil sociodemográfico era: o sexo masculino $(25 \%)$ e feminino $(75 \%)$, solteiros $(25 \%)$, casados $(62.5 \%)$ e divorciado (12.5\%), católicos $(75 \%)$ e evangélicos (25\%). Quanto ao nível de escolaridade a grande maioria dos entrevistados relatou possuía ensino fundamental incompleto $(87,5 \%)$ e um relatou não ser alfabetizado (12,5\%). Com idade média de $39 \pm 13,93$ anos. Em relação ao grau de parentesco dos entrevistados evidenciou-se que eram filhos $(37,5 \%)$, esposas $(37,5 \%)$, tia $(12,5 \%)$ e cuidadora (12,5\%).

As respostas qualitativas foram agrupadas nas categorias: O impacto da doença fora da possibilidade terapêutica de cura no contexto familiar e Importância da humanização e comunicação na assistência do enfermeiro.

\section{Categoria A - 0 impacto da doença fora da pos- sibilidade terapêutica de cura no contexto familiar}

O estado de saúde deteriorado acarreta em desequilíbrios da família, que são potencializados pela gravidade da doença. Estar internado em um ambiente hospitalar com atmosfera triste e impessoal, muda 
a rotina por completo, tudo passa a ser regrado e controlado, acaba por aumentar o medo, angústia, ansiedade e vulnerabilidade do doente e familiar, principalmente quando a cura é inexistente.

É praticamente impossível cuidar do indivíduo de forma integral, sem considerar seu contexto e relacionamento familiar, uma vez que o universo íntimo costuma ser abalado devido à doença crônica em fase terminal, ou seja, ao câncer e suas consequências emocionais, como também à grande oscilação que a terminalidade impõe, principalmente quando se esta sob cuidados paliativos de saúde.

A família acompanha e faz parte do processo do cuidado, tornando-se o único suporte para o doente. Quando falaram sobre o impacto da patologia fora das possibilidades terapêuticas, preocuparam-se em revelar os sentimentos existentes, trouxeram à angústia, ansiedade e conflitos como pontos de impacto da doença.

\section{A1 - Revelando a angústia e convivendo com a ansiedade}

Nos relatos das famílias, fica evidente que estas atravessam um momento delicado, com sentimentos confusos e permeados de incertezas, à medida que revelam a preocupação ao receber o diagnóstico.

[...] a gente fica nervoso, porque não quer perder.... e sabe que via perder um ente querido.... fico ansioso, e até agora não sei qual vai ser minha reação no momento que acontecer... não posso dizer que estou preparado porque não estou... (E 01).

É evidente que os familiares atravessam um momento delicado, confuso e incerto. Os profissionais devem saber quem são os familiares do paciente, tentar resgatar o entendimento que trazem da internação e esclarecer dúvidas, compartilhando dos sentimentos apresentados nesse momento, humanizando a assistência realizada.

A interação do enfermeiro com familiares é capaz de captar mensagens verbais, guardar e refletir sobre pontos relevantes do relato, para por meio dis- so, perceber sentimentos envolvidos no contexto em que a comunicação aconteceu. 0 conhecimento dos sentimentos da situação vivida permitiu realizar a interpretação, explicação e especificações dos dados colhidos, bem como compreender o turbilhão emocional que acomete familiares envolvidos no cuidar do paciente terminal.

A valorização do lado emocional, dos aspectos sociais e psicológicos do binômio paciente/família, consegue-se produzir uma maior qualidade à assistência, uma vez que o bom relacionamento e apoio promovem uma maior adaptação ao tratamento. Assim, a enfermagem paliativa corresponde ao cuidado prestado por meio da assistência integral, individual e contínua. Com relação à notícia do diagnóstico de câncer em estágio terminal, os familiares enfatizam a tristeza, medo e ansiedade vivida diante da doença sem possibilidades terapêuticas, que pode se observar:

\begin{abstract}
[...] a gente nunca espera... mais a gente sabe que ninguém tá livre dessas coisas, fiquei muito triste, abalado... (E 02).

Todo mundo ficou triste..., porque ter um pai e saber que ele não tem mais cura é difícil pra família... (E 03).

Pra a gente foi um choque, porque foi de repente... e ninguém sabia desse problema, então para família é um choque... (E 04).
\end{abstract}

No discurso fica evidente o caos emocional que a gravidade clínica produz. O enfermeiro deve aprender a trabalhar com a dor física e emocional presente quando se está trabalhando com pacientes terminais.

\section{A2 - Identificando os conflitos}

A individualidade dos diferentes componentes das linhagens pode interferir na relação familiar e potencializar os conflitos, uma vez que nem todos se comprometem do mesmo modo com o cuidado do paciente, deixam seus interesses pessoais prevalecer sobre os da família. Essa tendência pode gerar situações de atrito, ocasionadas pelo comportamento individual e egoísta dos envolvidos. 
Os resultados evidenciam que nem todos os familiares se envolvem no contexto do cuidar, deixam a cargo de alguns componentes da família a responsabilidade por acompanhar a internação e a convivência com os transtornos gerados pela patologia. Essa falta de envolvimento de toda a família, sobrecarrega cuidadores e abala as relações entre os membros, potencializa os atritos e conflitos dentro do contexto familiar. Ao observar-se as falas, percebe-se a presença dos conflitos.

Os familiares internaram ele aí. Depois vendo que estava agravando deixaram ele sozinho, foi quando a sobrinha dele tomou a frente para tomar conta dele... (E 08).

[...] ficou um relacionamento familiar abalado, por que eu tenho oito irmãos, dos oito só participam três... os outros tiraram o corpo... é muito difícil porque todo mundo tem suas obrigações, trabalho... mas abandonei tudo pra cuidar do meu pai. (E 05 ).

\section{Categoria B - Importância da humanização e co- municação na assistência do enfermeiro}

O conteúdo das entrevistas com os familiares permitiu identificar as facetas da comunicação adequada com o enfermeiro como ainda a fragilidade da assistência fundamentada nos princípios da humanização. Estas questões referem-se ao reconhecimento, por parte dos familiares, da figura do enfermeiro, do atendimento prestado e ao modo como foi atendido por este profissional.

Entre os dados encontrados, estão os referentes ao relacionamento entre a equipe de enfermagem e os usuários dos serviços de saúde representados pelas famílias entrevistadas. Entrevista apontou desconforto, descrito a seguir, que poderiam ser contornados com o auxílio da comunicação e da humanização do cuidado.

Eu acho que é obrigação deles, (dos enfermeiros) tem que ser obrigação deles fazer o que tem que ser feito pelo paciente. Não é a gente que tem que fazer não... tem momentos que quando o soro acaba o enfermeiro manda eu fechar o soro e depois, muito depois ele vêm... não gosto disso. (E 04).
B1 - Quando a comunicação e a humanização fazem parte da assistência do enfermeiro

Dados da pesquisa revelam que a maioria dos familiares não aprova a assistência prestada pelo enfermeiro e apontam falhas no processo de comunicação. Percebeu-se nas falas de poucos familiares, que a prática da humanização e comunicação entre enfermeiro/família, quando existem, produzem resultados positivos. Uma vez que, a orientação ao paciente e família pode proporcionar autonomia e diminuir a ansiedade e medo do desconhecido, conforme pode ser observado nos discursos.

[...] todos aqui são atenciosos, atendem bem os pacientes, muito bom, muito atencioso... todo mundo gosta desse enfermeiro. (E 03).

[...] a enfermeira chefe esta sempre presente quando a gente precisa... explica as coisas tudo direitinho é atenciosa e dedicada. (E 08).

No que concerne ao desenvolvimento da assistência integral, holística, humanizada e fortalecida pela comunicação, percebe-se que para as famílias, existe uma lacuna na assistência do enfermeiro. As falas revelam que a grande maioria não sabe identificar o enfermeiro na equipe de enfermagem, que sugere um desvio na comunicação e um desencontro com a valorização dos profissionais, na relação enfermeiro-paciente e enfermeiro-família. A ausência de orientações pode influenciar de forma negativa, interferir na continuidade do cuidado, bem como aumentar a ansiedade do paciente e família. Portanto, a dificuldade em compreender o processo de saúde e doença, bem como toda a assistência que é recebida gera insatisfação com o cuidado recebido. "[...] não sei por que eles entram, fazem o diagnóstico, botam o remédio e dão isso ou aquilo, mais eles não se identificam, nem falam o que vêm fazer..." (E04).

No tocante ao atendimento e orientações de enfermagem, as falas dos entrevistados evidenciaram um déficit na comunicação e humanização da assistência por parte do profissional enfermeiro. "Não, de 
maneira nenhuma, o que a gente faz é por experiência que nós temos, que a gente já vinha cuidando de meu pai em casa..." (E01).

\section{DISCUSSÃO}

A família é um sistema interpessoal formado por pessoas que possuem ligação por motivos diversos, assumem formas e tarefas que são criadas a partir de crenças, cultura, valores e normas (OLIVEIRA et al., 2014), representa vulnerabilidade e força, influencia na forma de ser e viver dos envolvidos (RODRIGUES; FERREIRA; MENEZES, 2010).

As neoplasias impactam sobre o paciente e família, alteram o contexto econômico, social, emocional, espiritual e de saúde. A morte é complexa de difícil aceitação, gera medo, angústia e conflitos, que abalam os indivíduos que estão ao seu alcance, intimida e causa desconforto nos envolvidos durante o processo (VARGAS et al., 2013).

Assistência de enfermagem ao paciente oncológico e a família deve ser pautada na comunicação e no relacionamento interpessoal. 0 convívio familiar é alterado pelo câncer, provoca alterações emocionais e oscilações, principalmente, quando se está sob cuidados paliativos (BUSHATSKY et al., 2012). 0 enfermeiro deve ter a sensibilidade para identificar as necessidades produzidas pela doença e atender de forma integral aos envolvidos no processo da doença (OLIVEIRA et al., 2014).

Os entrevistados reconheceram o papel do profissional Enfermeiro. Entretanto, relatam lacunas na comunicação e humanização da assistência ao paciente e família, que por vezes não são inseridos no plano de cuidado da enfermagem. Ainda se evidenciou o déficit na apresentação do enfermeiro, orientações à saúde e explicações sobre o cuidado prestado. Um estudo identificou que fatores como o carinho, o sorriso, a simpatia e a atenção contribuem para a humanização, enquanto que o mau humor, o barulho e o não ser atendido prontamente, dificultam esse processo (BRITO; CARVALHO, 2010).
A comunicação é essencial para a assistência, uma vez que ocorrem alterações significativas no contexto da doença terminal (FONTES; ALVIM, 2008), a interação enfermeiro-paciente e enfermeiro-família proporciona resultados positivos na assistência prestada (MARQUES; LARANJA; SILVA, 2014). Destaca-se que quando essa é efetiva, presta-se uma assistência de qualidade, de forma holística e resolutiva (RODRIGUES; FERREIRA; MENEZES, 2010), proporciona o levantamento das necessidades de saúde e possibilita que o enfermeiro elabore metas e ações para resolvê-Las ou amenizá-las (RENNÓ; CAMPOS, 2014).

Ressalta-se que as orientações, explicações, apresentação pessoal fazem parte do processo de comunicação e facilitam o melhor relacionamento terapêutico (RENNÓ; CAMPOS, 2014; RODRIGUES; FERREIRA; MENEZES, 2010). Está presente em todas as atividades do enfermeiro, tais como: entrevista, exame físico, planejamento da assistência, anotações nos prontuários, orientações aos indivíduos e família. Logo, constitui-se ferramenta essencial de todas as ações, influi na qualidade da assistência prestada àqueles que necessitam dos seus cuidados (LEITE; VASCONCELOS; FONTES, 2010).

No tocante a humanização da assistência, as falas dos familiares demostram no item anterior, minimização desse processo, uma vez que as atividades de enfermagem eram pautadas no tecnicismo e rotina. Existia subvalorização das alterações complexas e abrangentes que o câncer ocasiona. Humanizar a assistência é utilizar de recursos reconhecidos cientificamente para promover uma assistência de qualidade (BRITO; CARVALHO, 2010).

A humanização é conceituada como a valorização dos diferentes sujeitos envolvidos no processo de produção de saúde, com o estabelecimento de vínculos solidários, promove mudanças nos modelos de atenção e gestão dos processos de trabalho, tem como foco as necessidades dos cidadãos (BRASIL, 2005). Requer um processo reflexivo acerca dos valores e princípios que norteiam a prática profissional, além de proporcionar tratamento e cuidados dignos e solidários. Assistência humanizada é uma preocupação 
da enfermagem desde o século XIX. No Brasil, Wanda Horta, apresentou um modelo que permitiu o autocuidado, sem separar paciente e família durante a assistência de enfermagem (BRITO; CARVALHO, 2010).

A comunicação e assistência humanizada ao doente com câncer constitui um mecanismo para promover a qualidade do cuidado, que permite expressar os sentimentos, por meio das percepções, detectar e tratar áreas potencialmente problemáticas. Quando associada à terminalidade, auxilia a família e paciente, a identificar e expressar com a equipe, fatores relevantes nessa fase (KOVÁCS, 2009).

Uma abordagem humanista e integrada para o tratamento de pacientes sem possibilidade de cura pode reduzir sintomas e aumentar a qualidade de vida dos pacientes terminais (SILVA, 2006). 0 presente estudo demonstrou, na percepção de alguns familiares, lacunas na comunicação do enfermeiro. Outros consideraram positiva a relação comunicativa e humanizada frente à assistência do paciente e do núcleo familiar, o que fortalece a necessidade utilizar essas ferramentas no processo cuidar.

Dessa forma, a enfermagem paliativa corresponde à assistência integral, individualizada e contínua. Composta por ações de caráter técnico, científico e espiritual. Assim, as ferramentas assistências, são fortalecidas por meio da humanização do cuidado e da comunicação estruturada (FIGUEIREDO, 2006; MORITZ, 2007).

\section{CONCLUSÃO}

Ao identificar o processo de comunicação e humanização, bem como analisar na percepção da família de pacientes oncológicos, como o cuidado é compreendido, percebeu-se que família dos pacientes desconhece a figura do profissional enfermeiro, pois não o distingue dos demais profissionais de enfermagem. Existe a necessidade contínua, de valorização do ser enfermeiro, no sentido de mostrar o diferencial da atuação deste profissional para uma assistência que produz resultados significativos à saúde, para isso é necessário que se aproxime e reforce as atribuições nos padrões éticos, humanos e assistenciais.

Torna-se indispensável, programar ações de enfermagem humanizadas, a fim de auxiliar aos pacientes com doenças terminais e assim ofertar conforto e atendimento digno, por meio da valorização do emocional, dos aspectos sociais e psicológicos do binômio paciente/família. Assim, espera-se melhorar o relacionamento interpessoal, produzir uma assistência de qualidade e promover a adaptação ao tratamento.

\section{REFERÊNCIAS}

BRASIL. Ministério da Saúde. Conselho Nacional de Secretários de Saúde. CONASS. Política Nacional de Atenção Oncológica. Brasília, 2005. 9p.

BRASIL. Ministério da Saúde. Instituto Nacional de Câncer José Alencar Gomes da Silva. Estimativa 2016. Incidência do Câncer no Brasil. Rio de Janeiro: INCA, 2015.

BRITO, N.T.G.; CARVALHO, R. Humanization according to cancer patients with extended hospitalization periods. Einstein (São Paulo), São Paulo, v.8, n.2, p.221-7, 2010.

BUSHATSKY, M. et al. Cuidados Paliativos em pacientes fora de possibilidade terapêutica: um desafio para profissionais de saúde e cuidadores.

Rev. Centro Universitário São Camilo, São Camilo, v.34, n.65, p.399-408, 2012.

FIGUEIREDO, M.T.A. Reflexões sobre os cuidados paliativos no Brasil. Rev. Prática Hospitalar, São Paulo, v.8, n.47, p.36-40, 2006.

FONTES, C.A.S.; ALVIM, N.A.T. Importância do diálogo da enfermeira com clientes oncológicos diante do impacto do diagnóstico da doença. Cienc. Cuid. Saúde, Maringá, v.3, n.7, p.346-54, 2008. 
KOVÁCS, M.J. Comunicação em cuidados paliativos. In: PIMENTA, C.A.M; MOTA, D.D.C; CRUZ, D.A.L.M. (Eds.) Dor e cuidados paliativos. Barueri: Manole, 2009.

LEITE, N.C.; VASCONCELOS, J.M.B.; FONTES, W.D. A comunicação no Processo de Humanização da Assistência em Unidade de Terapia Intensiva: Vivência de Familiares e Cuidadores. Rev. enferm. UFPE on line, Recife, v.4, n.4, p.1587-1594, 2010.

MARQUES, D.L.L.; LARANJA, C.O.L.; SILVA, M.C.M. Interação entre família e equipe de enfermagem: repercussões na terapêutica do paciente oncológico.

Rev. enferm. UFPE [on-line], Recife, v.8, n.8, p.28112815, 2014.

MORITZ, R.D. Como melhorar a comunicação e prevenir conflitos nas situações de terminalidade na unidade de terapia intensiva. Rev. Bras. Ter. Intensiva, São Paulo, v.19, n.4, p.485-489, 2007.

OLIVEIRA, M.M. et al. Apreensões da família que cuida do seu familiar com câncer no domicílio. Rev. enferm. UFPE [on-line], Recife, v.8, n.4, p.827-833, 2014.

PETERSON, A.A.; CARVALHO, E.C. Comunicação terapêutica na Enfermagem: dificuldades para o cuidar de idosos com câncer. Rev. Bras. Enferm., Brasilia, v.4, n.64, p.692-697, 2011.
PINTO, M.H. et al. O cuidado de enfermagem ao paciente oncológico fora de possibilidade de cura: percepção de um grupo de profissionais. Cogitare Enferm., Curitiba, v.4, n.16, p.647-653, 2011.

RENNÓ, C.S.N.; CAMPOS, C.J.G. Comunicação interpessoal: valorização pelo paciente oncológico em uma unidade de alta complexidade em oncologia. Rev. Min. Enferm., Belo Horizonte, v.1, n.18, p.106115, 2014.

RODRIGUES, M.V.C.; FERREIRA, E.D.; MENEZES, T.M.O. Comunicação da enfermeira com pacientes portadores de câncer fora de possibilidade de cura.

Rev. enferm. UERJ, Rio de Janeiro, v.1, n.18, p.86-91, 2010.

\section{SILVA, M.J.P. Comunicação tem remédio: a}

comunicação nas relações interpessoais em saúde. São Paulo: Gente, 2006.

SILVA, M.M. et al. Análise do cuidado de enfermagem e da participação dos familiares na atenção paliativa oncológica. Texto Contexto Enferm., Florianópolis, v.3, n.21, p.658-666. 2012.

VARGAS, M.A.O. et al. Ressignificando o cuidado em uma unidade especializada em cuidados paliativos: uma realidade possível? Texto Contexto Enferm., Florianópolis, v.3, n.22, p.637-645. 2013. 
1 Graduado em Enfermagem pela Universidade Tiradentes - UNIT. Email: sebah_1@hotmail.com

2 Graduada em Enfermagem pela Universidade Tiradentes - UNIT. Email: heline@mblogistica-se.com.br

3 Mestrando em Biologia Parasitária pela Universidade Federal de Sergipe - UFS, São Cristóvão-SE. Email: damiao.arauj092@gmail.com

4 Profa. Me. do Departamento de Enfermagem da Universidade Federal de Sergipe - UFS, Campus de Lagarto-SE. Email: fernandagmsoares@gmail. com

5 Doutora em Enfermagem pela Universidade de São Paulo - USP. Email: inesbbocardi@yahoo.com.br

6 Profa. Me. do Departamento de Enfermagem da Universidade Federal de Sergipe - UFS, Campus de Lagarto-SE. Email: andreiacentenaro@ufs.br 